\title{
Metastatic breast cancer: the potential of miRNA for diagnosis and treatment monitoring
}

\author{
Andrew McGuire • James A. L. Brown • Michael J. Kerin
}

Published online: 27 February 2015

(C) The Author(s) 2015. This article is published with open access at Springerlink.com

\begin{abstract}
Breast cancer affects approximately $12 \%$ women worldwide and results in $14 \%$ of all cancer-related fatalities. Breast cancer is commonly categorized into one of four main subtypes (luminal A, luminal B, human epidermal growth factor receptor 2 (HER2) positive and basal), indicating molecular characteristics and informing treatment regimes. The most severe form of breast cancer is metastasis, when the tumour spreads from the breast tissue to other parts of the body. Significantly, the primary tumour subtype affects rates and sites of metastasis. Currently, up to $5 \%$ of patients present with incurable metastasis, with an additional $10-15 \%$ of patients going on to develop metastasis within 3 years of diagnosis. MicroRNAs (miRNAs) are short 21-25 long nucleotides that have been shown to significantly affect gene expression. Currently, $>2000$ miRNAs have been identified and significantly, specific miRNAs have been found associated with diseases states. Importantly, miRNAs are found circulating in the blood, presenting an opportunity to use these circulating disease-related miRNAs as biomarkers. Clearly, the identification of circulating miRNA specific to metastatic breast cancer presents a unique opportunity for early disease identification and for monitoring disease burden. Currently however, few groups have identified miRNA associated with metastatic breast cancer. Here, we review the literature surrounding the identification of metastatic miRNA in breast cancer patients, highlighting key areas where miRNA biomarker discovery could be beneficial, identifying key concepts, recognizing critical areas requiring further research and discussing potential problems.
\end{abstract}

ORCID: 0000-0002-3155-0334 (James A. L. Brown)

A. McGuire · J. A. L. Brown $(\bowtie) \cdot$ M. J. Kerin $(\bowtie)$

Discipline of Surgery, School of Medicine, National University of

Ireland, Galway, Ireland

e-mail: james.brown@nuigalway.ie

e-mail: michael.kerin@nuigalway.ie
Keywords miRNA · Breast cancer · Metastatic - Biomarker · Metastatic sites $\cdot$ Metastatic rates $\cdot$ Subtype

\section{Introduction}

Breast cancer is the second most common cancer diagnosed worldwide, affecting approximately one in eight women during their lifetime [1]. It affects 1.3 million women each year and accounts for $23 \%$ of all cancer cases and $14 \%(465,000)$ of all cancer-related deaths [2]. The most severe form of breast cancer occurs when the cancer spreads from the breast tissue to other regions of the body (metastasis), significantly increasing the tumour burden and often resulting in a fatal diagnosis. Breast cancer metastasis follows a cascade starting with local invasion of the surrounding tissue, spreading into the blood or lymphatic vessels and ending with dissemination of tumour cells to distal organs [3, 4] (Fig. 1, left). Despite modern treatments, metastatic breast cancer (MBC) is often incurable, with up to $5 \%$ of patients presenting with distal metastases at time of diagnosis [2]. Currently, distal metastasis (M1) occurs in $10-15 \%$ of patients within the first 3 years. Furthermore, approximately one third of women who have breast cancer with no lymph node involvement at time of diagnosis will develop distal metastases [5]. Significantly, the rate and site of metastasis can vary largely and is thought to be dependent on primary tumour subtype. Clearly, further knowledge is needed to both diagnose and treat metastatic breast cancer. Recently, microRNAs (miRNAs) have shown promise as new biomarkers for many cancers, including metastatic breast cancer [6-8]. Importantly, miRNAs have been linked to all stages along the metastatic cascade in breast cancer [9-15] (Fig. 1, right). Here we examine studies using circulating miRNAs as biomarkers for metastases, markers for tumour 
Fig. 1 Stages of breast cancer metastasis. Left: Order of process resulting in breast cancer metastasis. Right: Circulating miRNA associated with key steps in the metastatic cascade

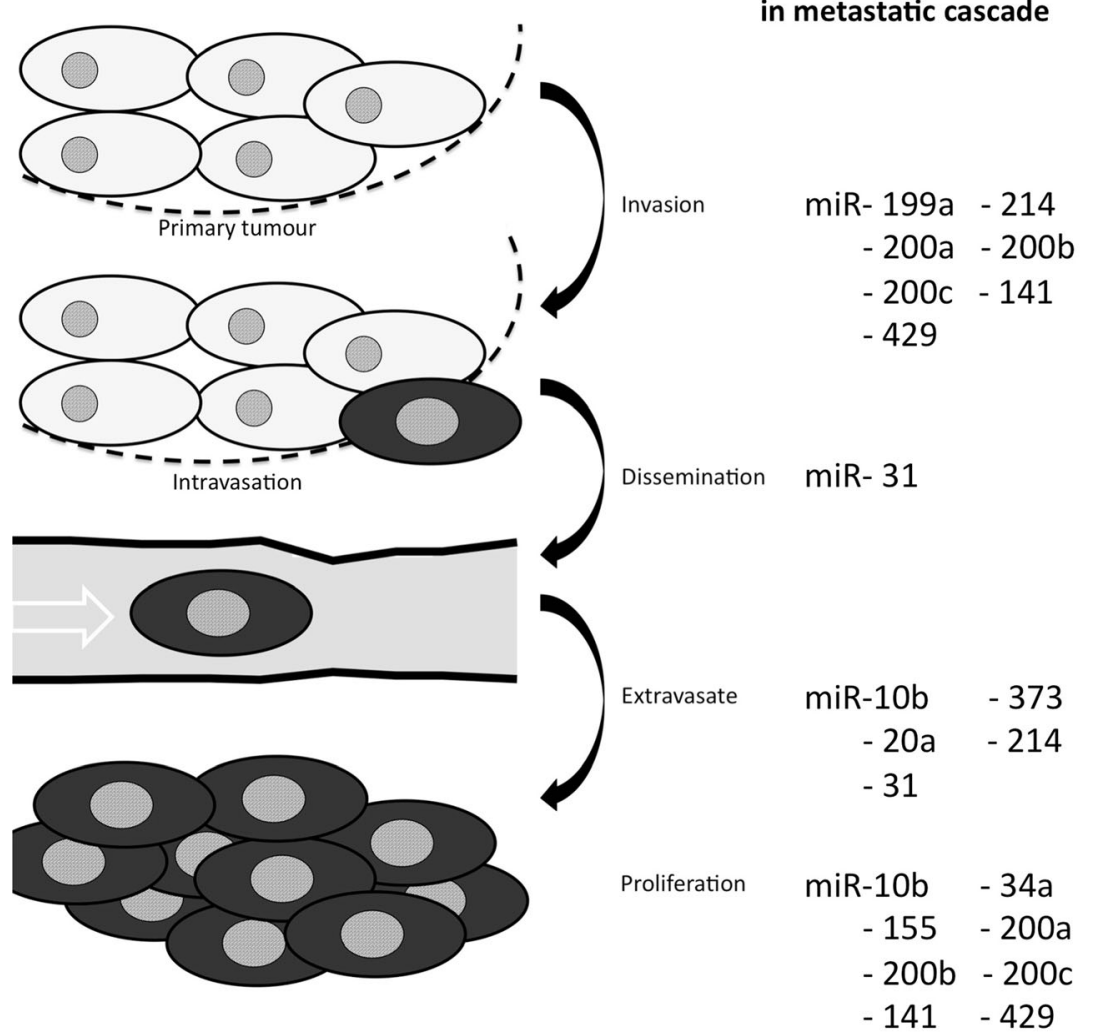

recurrence and response to clinical treatments. We likewise discuss the potential applications of miRNA for therapeutic metastatic breast cancer diagnosis, treatment and basic research.

\section{MicroRNA}

MicroRNAs (or miRNAs) were originally discovered in the early nineties in Caenorhabditis Elegans [16]. MicroRNAs are a 21-25 long class of small non-protein coding RNA that function as gene regulators by inhibiting the degradation of their target mRNAs and inhibiting translation (Fig. 2). miRNAs have been demonstrated to be involved in cell development, differentiation, proliferation and apoptosis [16]. The first human, disease-related miRNA characterized was from chronic lymphocytic leukemia [17] and subsequently, circulating miRNA were identified in patients with diffuse large B-cell lymphoma [18]. Consequently, miRNAs were linked to many other diseases and cancers [19, 20]. Since then, $>2000$ miRNAs have been identified in humans and these miRNAs regulate an estimated $30 \%$ of all human genes [21]. miRNA can exert their action in cancers through both tumour suppression and oncogenic mechanisms (as oncomirs) $[16,22]$. Fragile sites and genomic regions involved in oncogenic rearrangements in cancer are similarly thought to

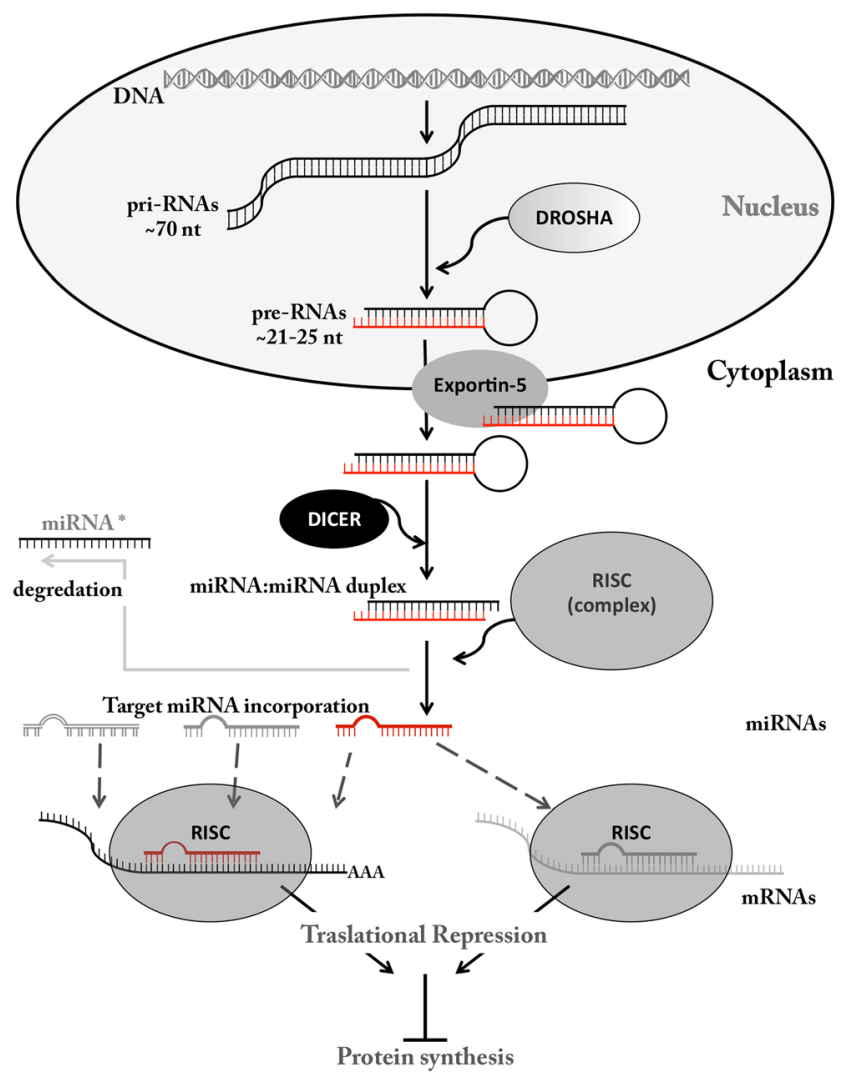

Fig. 2 miRNA biogenesis and mechanism of action 
influence the production of cancer-related miRNA [23]. Furthermore, as a proof-of-principle for any potential therapeutic application of miRNA, circulating miRNAs have been identified which correlate with breast cancer subtypes (Table 1) [24-27].

\section{1 miRNA biogenesis and action}

miRNAs are formed from precursors called pri-miRNAs that are processed in the nucleus by Drosha, an RNA III type nuclease. These pri-miRNAs are transported to the cytoplasm by exportin- 5 , where they are cleaved by Dicer, another RNAse III enzyme, forming an asymmetric duplex (miRNA:miRNA). This miRNA duplex is then separated, and the mature target miRNA molecule is incorporated into the RNA-induced silenced complex (RISC) where it binds a member of the Argonaute (Ago) protein family [the other miRNA molecule (miRNA*) is normally degraded] [16, 28, 29]. The active RISC complex is then able to target mRNA transcripts with a sequence complementary to the mature incorporated miRNA molecule, leading to inhibition of protein expression (Fig. 2). miRNA can be exported from cells packaged in membrane-bound extracellular compartments (exosomes) or bound to RNA binding proteins [30]. Exosomes provide another method of extracellular signalling as they are able to bind and merge with other cells, thus influencing their environment. Furthermore, exosomes have been directly implicated in cancer [31]. Significantly, miRNAs are differentially secreted or selectively packaged into exosomes, with different cell and tumour types displaying distinctive miRNA profiles [32].

\subsection{Breast cancer diagnosis}

Currently, breast cancer can be subcategorized based on the status (+/-) of the hormone receptors oestrogen receptor (ER) and progesterone receptor (PR) and the Receptor tyrosineprotein kinase erbB-2 (ERBB2 or HER2). Furthermore, recent genetic testing has enabled the molecular subtyping of breast cancers $[33,34]$. Presently, there are four major molecular subtypes: luminal A, $\sim 50-60 \%$ of breast cancers; luminal

Table 1 Breast cancer molecular subtypes

\begin{tabular}{llllll}
\hline $\begin{array}{l}\text { Breast cancer } \\
\text { subtype }\end{array}$ & \multicolumn{2}{l}{$\begin{array}{l}\text { Molecular } \\
\text { subtypes }\end{array}$} & & $\begin{array}{l}\text { Subtype-specific circulating } \\
\text { miRNA }\end{array}$ & Ref \\
\cline { 2 - 4 } & ER PR & HER2 & & \\
\hline Luminal A & + & + & - & miR-29a, miR-181a, miR- & {$[24]$} \\
& & & & 652 & miR-342 \\
Luminal B & + & + & + & miR-10b, miR-21 & {$[26]$} \\
HER2+ve & + & - & + & miR-210 & {$[27]$} \\
Basal (TNBC) & - & - & - & miR &
\end{tabular}

B, 10-20\%; HER $2+$ ve, $15-20 \%$, with the remaining $10-$ $20 \%$ considered Basal subtype [35]. Further subcategorizing the common molecular breast cancer subtypes has allowed clinicians to tailor treatments to each individual patients cancer [36]. In particular, the Oncotype DX test evaluates 16 cancer-related genes and 5 reference genes, with the results used to estimate the likely reoccurrence in patients and diagnostically to determine if a patient should receive chemotherapy [37]. Significantly, as miRNAs have been implicated in cancer metastasis, miRNA signatures are being pursued as novel clinical diagnostic targets to allow further subtyping of breast cancer and for predicting metastasis or therapeutic resistance [38-41]. The potential of miRNA as biomarker targets is facilitated by their stability in blood and their ability to withstand repeated freezing and thawing cycles [42].

\section{3 miRNA in metastatic breast cancer tissue}

Identifying and categorizing miRNAs expressed during the different stages of metastases will accelerate any therapeutic potential of these biomarkers, while illuminating the underlying mechanisms of cancer [43]. Currently, a number of studies have investigated miRNA expression profiles (upregulation or downregulation) of metastatic breast cancer tissue, providing insights into the processes of breast cancer initiation, progression and maintenance [44-47]. Significantly, it has been demonstrated that restoring the expression of individual miRNA observed to be lost in breast cancer models (such as miR-31, miR-126 or miR-335) can suppress metastasizes in vivo [48, 49]. Additionally, it has been suggested that cancer stem cells may influence metastasis [50-52], which would further contribute to any breast cancer miRNA profile. It is hoped that identifying breast cancer-specific miRNA and their functional relevance will lead to improvements in the early detection and treatment of tumours, particularly in younger patients.

\subsection{Metastases}

Breast cancer metastasises though the lymphatic system or via the circulatory system and is the overwhelming cause of mortality in patients with malignancies, causing $90 \%$ of deaths in solid tumours [53]. Metastasis in breast cancer is characterized by a distinctive spread via regional lymph nodes to the lungs, liver, brain and bones [54]. Importantly, the rates and sites of distal metastasis can vary depending on age and stage of diagnosis $[55,56]$. The most common site of metastases is the bone, often the first site of distal metastases in up to $50 \%$ of patients [57], with lungs and liver as the second and third most common metastatic sites (respectively) (Fig. 3a, left). Significantly, $10-15 \%$ of metastatic breast cancer patients will develop brain metastases, making breast cancer the second most common source of brain metastasis [58]. A similar distribution 


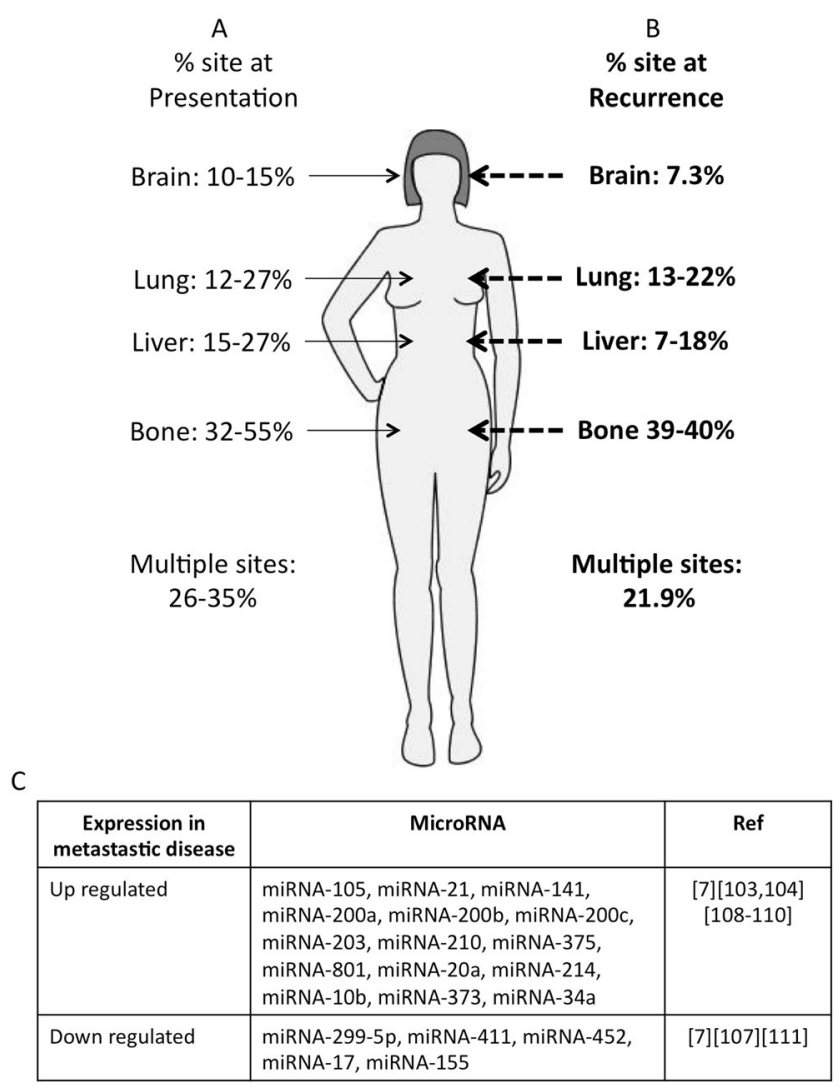

Fig. 3 a Sites of metastatic breast cancer at presentation/diagnosis. b Recurrence sites of metastatic breast cancer. c Summary of published miRNA-associated breast cancer

of metastasis is seen following relapse (post-treatment), with $\sim 22 \%$ of patients having multiple sites of metastasis (Fig. $3 \mathrm{~b}$, right) $[59,60]$.

Further examining metastasis by molecular breast cancer subtypes, distinctive patterns of metastasis sites are observed (Table 2). Bone metastases remain the most common metastatic site in luminal A, B and HER2+ve breast cancers [59, 61, 62]. However, basal cancers were found to primarily metastasize to the lungs [63]. Interestingly, luminal cancers also tend to have a lower rate of brain metastases. Significantly, brain metastasis for the HER2+ve subtype is high [64], despite the implementation of trastuzumab-based treatments for HER2+ ve breast cancers in the late 1990s. As Herceptin is not expected to cross the blood brain barrier, it is not believed to have

Table 2 Sites of breast cancer metastasis by molecular subtypes

\begin{tabular}{lllll} 
Metastasis sites & \multicolumn{3}{l}{ Breast cancer subtype } \\
\cline { 2 - 5 } & Luminal A & Luminal B & HER2+ve & Basal \\
\hline Brain & $6.6 \%$ & $8.2 \%$ & $23.3 \%$ & $18.1 \%$ \\
Lungs & $25.1 \%$ & $29.2 \%$ & $32.4 \%$ & $35.4 \%$ \\
Bone & $62.1 \%$ & $64.5 \%$ & $47.7 \%$ & $32.2 \%$ \\
Liver & $25.1 \%$ & $26 \%$ & $39.9 \%$ & $23.8 \%$ \\
\hline
\end{tabular}

influenced these rates. Currently, a number of circulating miRNA have been identified that are dysregulated (up or down regulated) in breast cancer metastasis (Fig. 3c). Identifying circulating miRNA associated with distinct metastatic sites could provide another powerful diagnostic tool for clinicians to evaluate disease stage and monitor progression.

\subsection{Subtype and metastasis stage}

Currently, the severity of a person's breast cancer is based on the TNM staging, where $\mathrm{T}$ describes the tumour size, $\mathrm{N}$ defines the lymph node status ( $+/-$ ) and M relates to any distant metastases $(0 / 1)$. In addition to TNM staging, breast cancer can be also divided into groups, stages $\mathrm{I}-\mathrm{V}$, depending on size and metastatic spread. Significantly, this combined staging is used clinically to inform the choice of treatment regime. However, TNM staging has its limitations and drawbacks, including overtesting and uncertainties in staging due to limits in sampling auxiliary lymph nodes. Furthermore, the four breast cancer molecular subtypes contain different disease progression, survival and relapse rates. Luminal subtypes tending to have slower metastatic spread, lower reoccurrence rates and better outcomes than HER2+ve or basal subtypes [61, 65-69]. This difference is independent of histological subtype or time of detection, with the majority of basal carcinomas detected in the early stages of breast cancer. When comparing median survival (from time of first distal metastasis), luminal A and B subtypes display longer overall survival (2.2 and 1.6 years) compared to HER2+ve subtype (1.3 years). However, the basal subtype has the worst overall survival rate ( 0.7 years). This is reflected in the presentation rates of the metastatic disease, stratified by molecular subtype (Table 3 ).

Significantly, the relapse rates vary considerably by subtype, with HER2+ve the highest (51.4\%), followed by luminal B (42.9 \%), basal (35.1\%) and luminal A (27.8 \%). Interestingly, in addition to the lowest recurrence rate, luminal A relapse also occurs later than the other subtypes [61]. Clinically, HER2+ve cancers have a poor prognosis; however, following development of anti-HER2 treatments, there has been an improvement in disease-free survival (from 72.2 to $78.6 \%$ ) $[70,71]$. Bone remains the most common primary metastatic site, while luminal B has a higher rate of metastases to other visceral organs (such as liver), compared to luminal

Table 3 Metastasis of breast cancer molecular subtypes (approximates)

\begin{tabular}{llll}
\hline $\begin{array}{l}\text { Breast cancer } \\
\text { subtype }\end{array}$ & $\begin{array}{l}\text { \% Metastasis at } \\
\text { presentation }\end{array}$ & $\begin{array}{l}\text { \% Metastasis at } \\
\text { recurrence }\end{array}$ & $\begin{array}{l}\text { MBC median } \\
\text { survival (years) }\end{array}$ \\
\hline Luminal A & $2-2.6$ & 27.8 & 2.2 \\
Luminal B & $1-2.5$ & 42.9 & 1.6 \\
HER2 & $5-6$ & 51.4 & 1.3 \\
Basal & $4-5$ & 35.1 & 0.7 \\
\hline
\end{tabular}


A. The basal subtype often presents with a younger onset, larger mean tumour size and higher grade, with the lowest overall survival $[72,73]$.

MicroRNAs have the potential to provide an additional mechanism for classifying breast cancer subtypes and tracking disease progression. A number of studies have investigated microRNAs in tissue as a means for identifying the main molecular breast cancer subtypes [24-26, 74-76]. Importantly, recent studies have found that microRNAs (miR-210, miR328 , miR-484 and miR-874) have the potential to predict prognosis or risk of recurrence $[26,77,78]$. Furthermore, it has been shown that microRNAs may be able to identify a subtype-specific response to treatment $[27,79,80]$.

\subsection{Breast cancer treatments}

Currently, surgery is the primary treatment for early stage breast cancer. However, the use of chemotherapy, radiotherapy and hormone therapy has vastly improved survival rates [81-83]. For the treatment of metastatic breast cancer, chemotherapy and radiotherapy are used in the neoadjuvant setting, before breast conservative surgery or mastectomy and axillary node clearance. In progressive disease (stage IV), chemotherapy and radiotherapy are the principal treatments, along with hormone therapy. Defining metastatic specific miRNA has the potential to categorize breast cancer and inform and improve treatment choices. Furthermore, the use of specific miRNA as therapeutics has the potential to one day become a valid treatment option [84-86]. Indeed, there are current clinical trials investigating the efficacy of using miRNA to treat cancer [87].

\subsection{Chemotherapy and miRNA}

Chemotherapy usually involves a combination of drugs and is the leading treatment, often combined with hormone therapy, in metastatic breast cancer. The most common chemotherapeutics used are anthracyclines (doxorubicin and epirubicin), taxanes (paclitaxel and docetaxel), fluorouracil (5-FU) and cyclophosphamide. Currently, there is no evidence of benefit of one regime over another. However, a meta-analysis has indicated a benefit of adding taxanes to an anthracyclinebased regime, demonstrating a 5 year risk reduction of $5 \%$ in disease-free survival and $3 \%$ in overall survival [88]. Significantly, HER2+ve patients treated with trastuzumab in combination with chemotherapy had increased median survival rates from 20.3 to 25.1 months [89]. Despite these treatment advancements, a large proportion of patients do not respond to traditional chemotherapy or hormone therapy [90]. In this context, circulating miRNAs have been explored as potential biomarkers, to predict treatment response [91-96]. Currently, only a few recent studies have explored the relationship of miRNAs with subtype-specific treatment [94, 97, 98] (Table 4). The early identification of circulating miRNA that can diagnose disease and/or chemotherapeutic responses will greatly facilitate improved treatments, leading to better outcomes for patients.

\section{Diagnosing metastatic disease}

Mammography is the gold standard for breast cancer screening, but it is mainly used for detection of local disease and is unreliable for diagnosing metastatic disease, with a false positive rate of $\sim 50 \%$ (7-9\% of these patients require a biopsy) [99]. Sentinel lymph nodes are the first lymph node in a tumour bed that receives lymphatic drainage from the tumour tissues, and sentinel lymph node biopsy (SLNB) currently provides the most accurate diagnosis for metastatic disease [100]. Currently, SLNB is recommended for early breast cancer, without any clinical evidence of nodal involvement [101]. However, SLNB only diagnoses regional metastasis. If distal metastases are suspected, SLNB needs to be combined with additional techniques, such as imaging. The development of an accurate biomarker, such as circulating miRNA, to diagnose or predict metastatic spread, could negate/reduce the need for many patients to undergo invasive procedures or surgery.

\subsection{Circulating miRNA as biomarkers in metastatic breast cancer}

Identifying circulating miRNA to use as biomarkers for metastatic breast cancer is currently a key priority for many research groups (Table 5). The first miRNA shown to be highly expressed in metastatic breast cancer was miR-10b (using mouse and human cells), with a clinical correlation in primary breast carcinomas [111]. A subsequent study confirmed this, finding elevated miR-10b, miR-34a and miR-155 levels in patients with metastatic breast cancer [7]. Further supporting this, it was recently shown that miR-10b and miR-373 were increased in lymph node positive breast cancer [108]. Excitingly, a significant increase in circulating miR-10b and miR373 was demonstrated in lymph node positive patients, compared to patients with no nodal involvement or healthy controls. Differences in miRNA levels in lymph node positive patients were also observed in a subsequent study [106], where higher levels of miR-20a and miR-214 were found in lymph node positive patients, compared to lymph node negative patients. miR-210 was also identified as a potential marker for lymph node metastasis, however only in a small cohort [94]. Interestingly, miR-10b was identified as a potential biomarker for brain [109] and bone [110] metastases in breast cancer. Together however, these independent results cast doubt on the use of miR-10b as a metastatic specific marker. Furthermore, miR-299-5p and miR-411 were found to have significant differences in metastatic breast cancer patients, 
Table 4 Metastatic breast cancer treatment regimes by subtype

\begin{tabular}{|c|c|c|c|c|}
\hline Subtype & Circulating miRNA & Menopause & Node negative & Node positive \\
\hline \multirow[t]{2}{*}{ Luminal A } & \multirow[t]{2}{*}{ miR-19a, miR-205 } & Pre & Tamoxifen \pm chemotherapy & Chemotherapy+tamoxifen \pm ovarian ablation \\
\hline & & Post & Aromatase inhibitor $(\mathrm{AI})+$ tamoxifen \pm chemotherapy & Chemotherapy + AI with tamoxifen \\
\hline \multirow[t]{2}{*}{ Luminal B } & \multirow[t]{2}{*}{ N.D } & Pre & Tamoxifen + Herceptin \pm chemotherapy & Chemotherapy + Herceptin + tamoxifen \\
\hline & & Post & $\begin{array}{l}\text { AI with tamoxifen }+ \text { herceptin } \pm \\
\text { chemotherapy }\end{array}$ & Chemotherapy + herceptin + AI with tamoxifen \\
\hline \multirow[t]{2}{*}{ HER2 } & \multirow[t]{2}{*}{$\operatorname{miR}-210$} & Pre & Herceptin+chemotherapy & Herceptin+chemotherapy \\
\hline & & Post & Herceptin+chemotherapy & Herceptin+chemotherapy \\
\hline \multirow[t]{2}{*}{ Basal } & \multirow{2}{*}{$\begin{array}{l}\text { miR-27a, miR-30e, } \\
\text { miR-155, miR-493 }\end{array}$} & Pre & \pm Chemotherapy & Chemotherapy \\
\hline & & Post & \pm Chemotherapy & Chemotherapy \\
\hline
\end{tabular}

Bone disease adds denosumab, zoledronic acid or pamidronate to chemotherapy regime

with the additional miRs miR-215 and miR-452 of interest, without reaching statistical significance [105]. Additionally, miR-21 has also been identified as a marker for breast cancer and predictor of stage [103]. Recently, eight miRNAs (miR141, miR-200a, miR-200b, miR-200c, miR-203, miR-210, miR-375 and miR-801) were found to be significantly higher in patients with circulating tumour cells (CTC) [104]. In another study, higher levels of miR-105 were found in early onset breast cancers that metastasized, compared to cancer that did not [102]. This study also found that overexpression of miR-105 promoted metastasis in vivo. The miRNAs miR17 and miR-155 have been identified as potential differentiators between metastatic and non-metastatic breast cancer [107]. Supporting those studies, a number of these miRNAs have previously been identified as markers in metastatic triple negative breast cancer samples [79]. As the above publications did not specifically state the sites of metastases, this may partially account for the diverse miRNA identified. Importantly, these studies highlight the importance and potential application of circulating miRNA as biomarkers that can discriminate non-metastatic from metastatic breast cancer.

Additionally, miRNAs have been identified in metastatic breast cancer tissue samples [9-11, 48, 112-114]. While there is some correlation between circulating and tissue microRNAs, again a large diversity of miRNAs were identified. The number of individual non-overlapping miRNAs identified highlights the complexity of metastasis, staging and breast cancer subtype definition. This emphasizes the need for further comprehensive investigations using similar comparable experimental methodology with more defined/ improved breast cancer typing criteria.

\section{Circulating miRNA in other metastatic tumours}

While the identification of circulating miRNA in breast cancer is progressing rapidly, exploring miRNAs investigated in other (often related) metastatic cancers may inform current work in the breast cancer field. Significantly, many of the circulating miRNA observed in other metastatic diseases have likewise been observed in metastatic breast cancer studies.

\section{1 miRNA in metastatic colon cancer}

In metastatic colon cancer, a significant increase in miR-29a in early liver metastasis was found [117]. In addition, high serum levels of miR-200b showed significant correlation with lymph node and distal metastatic disease in colorectal cancers [118]. Interestingly, miR$200 \mathrm{~b}$ was identified as an independent predictor of tumour reoccurrence in colon cancer.

Furthermore, tumour reoccurrence in colon cancer was found to be predicted by a panel of six miRNAs (miR-15a, miR-103, miR-148a, miR-320a, miR-451 and miR-596) [115]. Recently in metastatic colon cancer, three miRNAs (miR-106a, miR-130b and miR-484) were found to be significantly overexpressed in patients not responding to first-line chemotherapy [116].

\section{2 miRNA in metastatic cervical cancer}

In cervical squamous cell carcinoma, a group of six miRNAs (miR-20a, miR-1246, miR-2392, miR-3147, miR-3162-5p, miR-4484) were found to identify lymph node metastasis [124]. Supporting this, miR-20a was found to be significantly increased in patients with lymph node positive cervical cancer, compared to both controls and patients with lymph node negative disease [122].

\section{3 miRNA in metastatic gastric cancer}

A recent study identified six miRNAs significantly increased in lymph node metastases of gastric cancer: miR-21, miR-27a, miR-106b, miR-146a, miR-148a and miR-223 [123]. In 
Table 5 MicroRNA in metastatic tumours

\begin{tabular}{|c|c|c|c|}
\hline MicroRNA & Tumour & Ref. & Cohort $(N)$ \\
\hline miRNA-105 & Breast (serum) & [102] & 38 Patients \\
\hline miRNA-21 & Breast (serum) & {$[103]$} & $\begin{array}{l}102 \text { Patients, } 20 \\
\text { controls }\end{array}$ \\
\hline $\begin{array}{l}\text { miRNA-141, miRNA-200a, miRNA-200b, miRNA-200c, } \\
\text { miRNA-203, miRNA-210, miRNA-375, miRNA-801 }\end{array}$ & Breast (serum) & {$[104]$} & $\begin{array}{l}61 \text { Patients, } 76 \\
\text { controls }\end{array}$ \\
\hline miRNA-215, miRNA-299-5p, miRNA-411, miRNA-452 & Breast (serum) & {$[105]$} & $\begin{array}{l}75 \text { Patients, } 20 \\
\text { controls }\end{array}$ \\
\hline miRNA-20a, miRNA-214 & Breast (serum) & [106] & $\begin{array}{l}48 \text { Patients, } 54 \\
\text { controls }\end{array}$ \\
\hline miRNA-210 & Breast (serum) & [94] & $\begin{array}{l}8 \text { Patients, } 31 \\
\text { controls }\end{array}$ \\
\hline miRNA-17, miRNA-155 & Breast (serum) & {$[107]$} & $\begin{array}{l}72 \text { Patients, } 40 \\
\text { controls }\end{array}$ \\
\hline miRNA-10b, miRNA-373 & Breast (serum) & [108] & $\begin{array}{l}35 \text { Patients, } 10 \\
\text { controls }\end{array}$ \\
\hline miRNA-10b, miRNA-34a, miRNA-155 & Breast (serum) & {$[7]$} & $\begin{array}{l}30 \text { Patients, } 29 \\
\text { controls }\end{array}$ \\
\hline miRNA-10b & Breast (serum) & [109] & $\begin{array}{l}20 \text { Patients, } 10 \\
\text { controls }\end{array}$ \\
\hline miRNA-10b & Breast (serum) & {$[110]$} & $\begin{array}{l}122 \text { Patients, } 59 \\
\text { controls }\end{array}$ \\
\hline miRNA-10b & Breast (serum) & [111] & 23 Patients \\
\hline miRNA-126, miRNA-335 & Breast (tissue) & {$[48]$} & 11 Patients \\
\hline miRNA-21, miRNA-139-5p, miRNA-486-5p & Breast (tissue) & {$[9]$} & 6 Patients \\
\hline $\begin{array}{l}\text { Let 7i, miRNA-16, miRNA-26a, miRNA-27a, miRNA-143, } \\
\text { miRNA-196a, miRNA-375, miRNA-503, miRNA-519a, } \\
\text { miRNA-519b-3q, miRNA-361-5p }\end{array}$ & Breast (tissue) & {$[10]$} & 48 Patients \\
\hline miRNA-27b-3q, miRNA-107, miRNA-103a-3p & Breast (tissue) & {$[11]$} & 58 Patients \\
\hline miRNA-22 & Breast (tissue) & [112] & 108 Patients \\
\hline miRNA-373 & Breast (tissue) & [113] & 11 Patients \\
\hline miRNA-21 & Breast (tissue) & {$[114]$} & 113 Patients \\
\hline $\begin{array}{l}\text { miRNA-15a, miRNA-103, miRNA-148a, miRNA-320a, } \\
\text { miRNA-451, miRNA-596 }\end{array}$ & Colon (serum) & [115] & 30 Patients \\
\hline miRNA-27b, miRNA-158a, miRNA-326 & Colon (serum) & {$[116]$} & 150 Patients \\
\hline miRNA-29a & Colon (serum) & [117] & 20 Patients \\
\hline miRNA-200c & Colon (serum) & [118] & 182 Patients \\
\hline miRNA-141 & Prostate (serum) & [119] & 21 Patients \\
\hline miRNA-141 & Prostate (serum) & {$[120]$} & 56 Patients \\
\hline miRNA-141, miRNA-375, miRNA-378 & Prostate (serum) & {$[121]$} & 84 Patients \\
\hline miRNA-20a, miRNA-203 & Cervical (serum) & {$[122]$} & 80 Patients \\
\hline $\begin{array}{l}\text { miRNA-21, miRNA-27a, miRNA-106b, miRNA-146a, } \\
\text { miRNA-148a, miRNA-223 }\end{array}$ & Gastric (serum) & [123] & $\begin{array}{l}20 \text { Controls, } 16 \\
\text { patients }\end{array}$ \\
\hline
\end{tabular}

addition, a significant increase in levels of miR-21, miR-146a and miR-148a were found to correlate with increased spread in the lymph node.

\section{4 miRNA in metastatic prostate cancer}

Recent work in metastatic prostate cancer found miR141 to accurately predict treatment response, compared to standard markers such as prostate-specific antigen
(PSA), lactate dehydrogenase and circulating tumour cells [119]. Interestingly, levels of miRA-141 were found to be elevated in bone metastatic prostate cancer [120]. Importantly, miR-141 expression levels were found to correlate to alkaline phosphatase but not to PSA. A further study looking at metastatic castration resistant prostate cancer again found miR-141, plus miR-375 and miR-378 to be overexpressed compared to low-risk localized patients [121]. 


\section{Conclusions}

Our understanding of metastatic breast cancer has advanced considerably over the last number of years, yet metastasis remains the major cause of morbidity and mortality in breast cancer. Up to one in three breast cancer patients diagnosed will develop metastatic breast cancer, and despite current treatments, $78 \%$ of these will die within 5 years. Clearly, there is an urgent need to find new clinically relevant biomarkers and tests to allow the early detection of metastatic breast cancer and for the monitoring of treatment response.

The defining of different molecular breast cancer subtypes has significantly aided the treatment of breast cancer, allowing more tailored individual treatment regimes. Significantly, treatment using hormone therapies and Herceptin has increased survival in luminal and HER2 positive breast cancers. However, basal (triple negative) breast cancers continue to have poorer outcomes. Chemotherapy remains the major treatment for metastatic breast cancer, yet similar regimes are given for all subtypes. Additional research is needed to define further subtypes and identify new markers that predict their response to chemotherapy. MicroRNAs have emerged as one such potential marker for predicting metastatic disease and response to treatment. Recently, a small number of miRNAs have shown increased expression in the circulation of metastatic breast cancer patients. In particular, miR-10b has been highlighted across five studies and has been linked to specific sites of distal metastasis. Despite the differences in the rate of metastasis across the breast cancer subtypes, to our knowledge, only one study (Dai et al.) has assessed the miRNA profiles associated with each molecular subtype [125]. Importantly, this study used tumour samples, not circulating miRNA, and did not include metastatic disease. A study investigating circulating miRNA profiles in patients with different molecular subtypes and metastatic disease is greatly needed. Furthermore, the use of different extraction methods and starting material may explain the lack of consensus between microRNAs currently identified in the indicated studies. However, another possible reason may be the diversity of sites of distal metastasis in each study. Only two studies have explored metastatic site-specific miRNAs, Ahmad et al. [109] (brain) and Zhao et al. [110] (bone), with both studies identifying elevated miR-10b. The identification of truly site- or subtype-specific metastatic miRNA may provide the diagnostic tool required to improve personalized metastatic breast cancer treatments. Interestingly, many circulating miRNAs identified in metastatic breast cancer were also found in studies of other metastatic cancers. This may indicate that the identified miRNAs are indicative of the sites of metastasis or that the miRNAs correspond to common underlying mechanisms of cancer metastasis.

Here, we highlighted the current knowledge and potential of microRNAs as biomarkers for improving the diagnosis and treatment of metastatic breast cancer. However, significant further directed research is needed to identify and confirm miRNA that can predict site-specific metastasis disease outcome or patient response to treatments.

Acknowledgments We would like to thank all the members of Prof Kerins' group for stimulating discussion. AM, JALB and MK are funded by the National Breast Cancer Research Institute (NBCRI).

Conflict of interest The authors declare that they have no conflict of interest.

Open Access This article is distributed under the terms of the Creative Commons Attribution License which permits any use, distribution, and reproduction in any medium, provided the original author(s) and the source are credited.

\section{References}

1. Siegel, R., Naishadham, D., \& Jemal, A. (2013). Cancer statistics, 2013. CA: A Cancer Journal for Clinicians, 63, 11.

2. Jemal, A., et al. (2011). Global cancer statistics. CA: A Cancer Journal for Clinicians, 61, 69.

3. Hunter, K. W., Crawford, N. P., \& Alsarraj, J. (2008). Mechanisms of metastasis. Breast Cancer Research, 10 (Suppl 1), S2.

4. Scully, O. J., Bay, B. H., Yip, G., \& Yu, Y. (2012). Breast cancer metastasis. Cancer Genomics Proteomics, 9, 311.

5. Weigelt, B., Peterse, J. L., \& van 't Veer, L. J. (2005). Breast cancer metastasis: markers and models. Nature Reviews Cancer, 5, 591.

6. Heneghan, H. M., et al. (2010). Circulating microRNAs as novel minimally invasive biomarkers for breast cancer. Annals of Surgery, $251,499$.

7. Roth, C., et al. (2010). Circulating microRNAs as blood-based markers for patients with primary and metastatic breast cancer. Breast Cancer Research, 12, R90.

8. Bartels, C. L., \& Tsongalis, G. J. (2009). MicroRNAs: novel biomarkers for human cancer. Clinical Chemistry, 55, 623.

9. Rask, L., et al. (2014). Differential expression of miR-139, miR-486 and miR-21 in breast cancer patients sub-classified according to lymph node status. Cellular Oncology (Dordr), 37, 215.

10. Smeets, A., et al. (2011). Prediction of lymph node involvement in breast cancer from primary tumor tissue using gene expression profiling and miRNAs. Breast Cancer Research and Treatment, 129, 767.

11. Shen, S., et al. (2014). A prognostic model of triple-negative breast cancer based on miR-27b-3p and node status. PloS One, 9, e100664.

12. Yu, F., et al. (2007). let-7 regulates self renewal and tumorigenicity of breast cancer cells. Cell, 131, 1109.

13. Ye, Z. B., et al. (2015). miR-429 inhibits migration and invasion of breast cancer cells in vitro. International Journal of Oncology, 46, 531.

14. S. M. Yahya, G. H. Elsayed (2014) A summary for molecular regulations of miRNAs in breast cancer. Clinical Biochemistry.

15. Dykxhoorn, D. M. (2010). MicroRNAs and metastasis: little RNAs go a long way. Cancer Research, 70, 6401.

16. Esquela-Kerscher, A., \& Slack, F. J. (2006). OncomirsmicroRNAs with a role in cancer. Nature Reviews Cancer, 6, 259.

17. Calin, G. A., et al. (2002). Frequent deletions and down-regulation of micro-RNA genes miR15 and miR16 at 13q14 in chronic lymphocytic leukemia. Proceedings of the National Academy of Sciences of the United States of America, 99, 15524. 
18. Lawrie, C. H., et al. (2008). Detection of elevated levels of tumourassociated microRNAs in serum of patients with diffuse large B-cell lymphoma. British Journal of Haematology, 141, 672.

19. Lujambio, A., \& Lowe, S. W. (2012). The microcosmos of cancer. Nature, 482, 347.

20. Croce, C. M., \& Calin, G. A. (2005). miRNAs, cancer, and stem cell division. Cell, 122, 6 .

21. Christodoulatos, G. S., \& Dalamaga, M. (2014). Micro-RNAs as clinical biomarkers and therapeutic targets in breast cancer: Quo vadis? World Journal of Clinical Oncology, 5, 71.

22. Kent, O. A., \& Mendell, J. T. (2006). A small piece in the cancer puzzle: microRNAs as tumor suppressors and oncogenes. Oncogene, 25, 6188.

23. Calin, G. A., et al. (2004). Human microRNA genes are frequently located at fragile sites and genomic regions involved in cancers. Proceedings of the National Academy of Sciences of the United States of America, 101, 2999.

24. McDermott, A. M., et al. (2014). Identification and validation of oncologic miRNA biomarkers for luminal A-like breast cancer. PloS One, 9, e87032.

25. Lowery, A. J., et al. (2009). MicroRNA signatures predict oestrogen receptor, progesterone receptor and HER2/neu receptor status in breast cancer. Breast Cancer Research, 11, R27.

26. Anfossi, S., et al. (2014). High serum miR-19a levels are associated with inflammatory breast cancer and are predictive of favorable clinical outcome in patients with metastatic HER2+ inflammatory breast cancer. PloS One, 9, e83113.

27. Toyama, T., et al. (2012). High expression of microRNA-210 is an independent factor indicating a poor prognosis in Japanese triplenegative breast cancer patients. Japanese Journal of Clinical Oncology, 42, 256.

28. Munker, R., \& Calin, G. A. (2011). MicroRNA profiling in cancer. Clinical Science (London), 121, 141.

29. He, L., \& Hannon, G. J. (2004). MicroRNAs: small RNAs with a big role in gene regulation. Nature Reviews Genetics, 5, 522.

30. Arroyo, J. D., et al. (2011). Argonaute2 complexes carry a population of circulating microRNAs independent of vesicles in human plasma. Proceedings of the National Academy of Sciences of the United States of America, 108, 5003.

31. Vella, L. J. (2014). The emerging role of exosomes in epithelialmesenchymal-transition in cancer. Frontiers in Oncology, 4, 361.

32. Lu, J., et al. (2005). MicroRNA expression profiles classify human cancers. Nature, 435, 834.

33. Perou, C. M., et al. (2000). Molecular portraits of human breast tumours. Nature, 406, 747.

34. Goldhirsch, A., et al. (2011). Strategies for subtypes - dealing with the diversity of breast cancer: highlights of the St. Gallen International Expert Consensus on the Primary Therapy of Early Breast Cancer 2011. Annals of Oncology, 22, 1736.

35. Eroles, P., Bosch, A., Perez-Fidalgo, J. A., \& Lluch, A. (2012). Molecular biology in breast cancer: intrinsic subtypes and signaling pathways. Cancer Treatment Reviews, 38, 698.

36. Eiermann, W., et al. (2013). The 21-gene recurrence score assay impacts adjuvant therapy recommendations for ER-positive, nodenegative and node-positive early breast cancer resulting in a riskadapted change in chemotherapy use. Annals of Oncology, 24, 618.

37. Dobbe, E., Gurney, K., Kiekow, S., Lafferty, J. S., \& Kolesar, J. M. (2008). Gene-expression assays: new tools to individualize treatment of early-stage breast cancer. American Journal of HealthSystem Pharmacy, 65, 23.

38. Baffa, R., et al. (2009). MicroRNA expression profiling of human metastatic cancers identifies cancer gene targets. Journal of Pathology, 219, 214.

39. Shi, M., Liu, D., Duan, H., Shen, B., \& Guo, N. (2010). Metastasisrelated miRNAs, active players in breast cancer invasion, and metastasis. Cancer and Metastasis Reviews, 29, 785.
40. Guttery, D. S., et al. (2013). Hide and seek: tell-tale signs of breast cancer lurking in the blood. Cancer and Metastasis Reviews, 32, 289.

41. Zhang, J., \& Ma, L. (2012). MicroRNA control of epithelialmesenchymal transition and metastasis. Cancer and Metastasis Reviews, 31, 653.

42. Mitchell, P. S., et al. (2008). Circulating microRNAs as stable blood-based markers for cancer detection. Proceedings of the National Academy of Sciences of the United States of America, 105, 10513.

43. Wang, L., \& Wang, J. (2012). MicroRNA-mediated breast cancer metastasis: from primary site to distant organs. Oncogene, 31, 2499.

44. Jang, K., et al. (2014). Loss of microRNA-200a expression correlates with tumor progression in breast cancer. Translational Research: The Journal of Laboratory and Clinical Medicine, 163, 242.

45. Markou, A., Yousef, G. M., Stathopoulos, E., Georgoulias, V., \& Lianidou, E. (2014). Prognostic significance of metastasis-related microRNAs in early breast cancer patients with a long follow-up. Clinical Chemistry, 60, 197.

46. Cascione, L., et al. (2013). Integrated microRNA and mRNA signatures associated with survival in triple negative breast cancer. PloS One, 8, e55910.

47. Hafez, M. M., et al. (2012). MicroRNAs and metastasis-related gene expression in Egyptian breast cancer patients. Asian Pacific Journal of Cancer Prevention: APJCP, 13, 591.

48. Tavazoie, S. F., et al. (2008). Endogenous human microRNAs that suppress breast cancer metastasis. Nature, 451, 147.

49. Valastyan, S., et al. (2009). A pleiotropically acting microRNA, miR-31, inhibits breast cancer metastasis. Cell, 137, 1032.

50. Li, F., Tiede, B., Massague, J., \& Kang, Y. (2007). Beyond tumorigenesis: cancer stem cells in metastasis. Cell Research, 17, 3.

51. Singh, S. K., et al. (2003). Identification of a cancer stem cell in human brain tumors. Cancer Research, 63, 5821.

52. Cuiffo, B. G., et al. (2014). MSC-Regulated MicroRNAs Converge on the Transcription Factor FOXP2 and Promote Breast Cancer Metastasis. Cell Stem Cell, 15, 762.

53. Gupta, G. P., \& Massague, J. (2006). Cancer metastasis: building a framework. Cell, 127, 679 .

54. Muller, A., et al. (2001). Involvement of chemokine receptors in breast cancer metastasis. Nature, 410, 50.

55. Ernst, M. F., et al. (2007). Trends in the prognosis of patients with primary metastatic breast cancer diagnosed between 1975 and 2002. Breast, 16, 344.

56. Ren, Z., Li, Y., Hameed, O., Siegal, G. P., \& Wei, S. (2014). Prognostic factors in patients with metastatic breast cancer at the time of diagnosis. Pathology Research and Practice, 210, 301.

57. Morris, P. G., et al. (2010). Integrated positron emission tomography/computed tomography may render bone scintigraphy unnecessary to investigate suspected metastatic breast cancer. Journal of Clinical Oncology, 28, 3154.

58. Barnholtz-Sloan, J. S., et al. (2004). Incidence proportions of brain metastases in patients diagnosed (1973 to 2001) in the Metropolitan Detroit Cancer Surveillance System. Journal of Clinical Oncology, 22, 2865.

59. Sihto, H., et al. (2011). Breast cancer biological subtypes and protein expression predict for the preferential distant metastasis sites: a nationwide cohort study. Breast Cancer Research, 13, R87.

60. Berman, A. T., Thukral, A. D., Hwang, W. T., Solin, L. J., \& Vapiwala, N. (2013). Incidence and patterns of distant metastases for patients with early-stage breast cancer after breast conservation treatment. Clinical Breast Cancer, 13, 88.

61. Kennecke, H., et al. (2010). Metastatic behavior of breast cancer subtypes. Journal of Clinical Oncology, 28, 3271. 
62. J. M. Matro et al. (2014) Inflammatory breast cancer management in the national comprehensive cancer network: the disease, recurrence pattern, and outcome. Clinical Breast Cancer.

63. Smid, M., et al. (2008). Subtypes of breast cancer show preferential site of relapse. Cancer Research, 68, 3108.

64. C. Aversa et al. (2014) Metastatic breast cancer subtypes and central nervous system metastases. Breast.

65. Lowery, A. J., Kell, M. R., Glynn, R. W., Kerin, M. J., \& Sweeney, K. J. (2012). Locoregional recurrence after breast cancer surgery: a systematic review by receptor phenotype. Breast Cancer Research and Treatment, 133, 831.

66. Bauer, K. R., Brown, M., Cress, R. D., Parise, C. A., \& Caggiano, V. (2007). Descriptive analysis of estrogen receptor (ER)-negative, progesterone receptor (PR)-negative, and HER2-negative invasive breast cancer, the so-called triple-negative phenotype: a populationbased study from the California cancer Registry. Cancer, 109, 1721.

67. Carey, L. A., et al. (2006). Race, breast cancer subtypes, and survival in the Carolina Breast Cancer Study. JAMA, 295, 2492.

68. Ihemelandu, C. U., et al. (2007). Molecular breast cancer subtypes in premenopausal and postmenopausal African-American women: age-specific prevalence and survival. Journal of Surgical Research, 143,109

69. Onitilo, A. A., Engel, J. M., Greenlee, R. T., \& Mukesh, B. N. (2009). Breast cancer subtypes based on ER/PR and Her2 expression: comparison of clinicopathologic features and survival. Clinical Medicine \& Research, 7, 4.

70. Piccart-Gebhart, M. J., et al. (2005). Trastuzumab after adjuvant chemotherapy in HER2-positive breast cancer. New England Journal of Medicine, 353, 1659.

71. Gianni, L., et al. (2011). Treatment with trastuzumab for 1 year after adjuvant chemotherapy in patients with HER2-positive early breast cancer: a 4-year follow-up of a randomised controlled trial. The Lancet Oncology, 12, 236.

72. Bosch, A., Eroles, P., Zaragoza, R., Vina, J. R., \& Lluch, A. (2010). Triple-negative breast cancer: molecular features, pathogenesis, treatment and current lines of research. Cancer Treatment Reviews, 36, 206.

73. Parker, J. S., et al. (2009). Supervised risk predictor of breast cancer based on intrinsic subtypes. Journal of Clinical Oncology, 27, 1160.

74. Blenkiron, C., et al. (2007). MicroRNA expression profiling of human breast cancer identifies new markers of tumor subtype. Genome Biology, 8, R214.

75. Enerly, E., et al. (2011). miRNA-mRNA integrated analysis reveals roles for miRNAs in primary breast tumors. PloS One, 6, e16915.

76. Janssen, E. A., et al. (2010). Biologic profiling of lymph node negative breast cancers by means of microRNA expression. Modern Pathology, 23, 1567.

77. Li, Y., et al. (2013). microRNA-210 as a prognostic factor in patients with breast cancer: meta-analysis. Cancer Biomarkers, 13, 471.

78. Volinia, S., \& Croce, C. M. (2013). Prognostic microRNA/mRNA signature from the integrated analysis of patients with invasive breast cancer. Proceedings of the National Academy of Sciences of the United States of America, 110, 7413.

79. Avery-Kiejda, K. A., Braye, S. G., Mathe, A., Forbes, J. F., \& Scott, R. J. (2014). Decreased expression of key tumour suppressor microRNAs is associated with lymph node metastases in triple negative breast cancer. BMC Cancer, 14, 51 .

80. Farazi, T. A., et al. (2011). MicroRNA sequence and expression analysis in breast tumors by deep sequencing. Cancer Research, 71,4443

81. Jacobson, J. A., et al. (1995). Ten-year results of a comparison of conservation with mastectomy in the treatment of stage I and II breast cancer. New England Journal of Medicine, 332, 907.

82. Fyles, A. W., et al. (2004). Tamoxifen with or without breast irradiation in women 50 years of age or older with early breast cancer. New England Journal of Medicine, 351, 963.
83. G. Early Breast Cancer Trialists' Collaborative. (2005). Effects of chemotherapy and hormonal therapy for early breast cancer on recurrence and 15-year survival: an overview of the randomised trials. Lancet, 365, 1687.

84. Rossi, M., et al. (2013). From target therapy to miRNA therapeutics of human multiple myeloma: theoretical and technological issues in the evolving scenario. Current Drug Targets, 14, 1144.

85. Pereira, D. M., Rodrigues, P. M., Borralho, P. M., \& Rodrigues, C. M. (2013). Delivering the promise of miRNA cancer therapeutics. Drug Discovery Today, 18, 282.

86. Uchino, K., Ochiya, T., \& Takeshita, F. (2013). RNAi therapeutics and applications of microRNAs in cancer treatment. Japanese Journal of Clinical Oncology, 43, 596.

87. Conde, J., \& Artzi, N. (2015). Are RNAi and miRNA therapeutics truly dead? Trends in Biotechnology(Jan 13).

88. De Laurentiis, M., et al. (2008). Taxane-based combinations as adjuvant chemotherapy of early breast cancer: a meta-analysis of randomized trials. Journal of Clinical Oncology, 26, 44.

89. Slamon, D. J., et al. (2001). Use of chemotherapy plus a monoclonal antibody against HER2 for metastatic breast cancer that overexpresses HER2. New England Journal of Medicine, 344, 783.

90. Zambetti, M., et al. (2012). Pathological complete response rates following different neoadjuvant chemotherapy regimens for operable breast cancer according to ER status, in two parallel, randomized phase II trials with an adaptive study design (ECTO II). Breast Cancer Research and Treatment, 132, 843.

91. Chen, J., Tian, W., Cai, H., He, H., \& Deng, Y. (2012). Downregulation of microRNA-200c is associated with drug resistance in human breast cancer. Medical Oncology, 29, 2527.

92. P. Freres et al. (2014) Neoadjuvant chemotherapy in breast cancer patients induces miR-34a and miR-122 expression. Journal of Cellular Physiology.

93. Hoppe, R., et al. (2013). Increased expression of miR-126 and miR10a predict prolonged relapse-free time of primary oestrogen receptor-positive breast cancer following tamoxifen treatment. European Journal of Cancer, 49, 3598.

94. Jung, E. J., et al. (2012). Plasma microRNA 210 levels correlate with sensitivity to trastuzumab and tumor presence in breast cancer patients. Cancer, 118, 2603.

95. Sun, Y., et al. (2012). Serum microRNA-155 as a potential biomarker to track disease in breast cancer. PloS One, 7, e47003.

96. Wang, H., et al. (2012). Circulating MiR-125b as a marker predicting chemoresistance in breast cancer. PloS One, 7, e34210.

97. Li, Q., et al. (2014). Circulating miR-19a and miR-205 in serum may predict the sensitivity of luminal A subtype of breast cancer patients to neoadjuvant chemotherapy with epirubicin plus paclitaxel. PloS One, 9, e104870.

98. Gasparini, P., et al. (2014). microRNA expression profiling identifies a four microRNA signature as a novel diagnostic and prognostic biomarker in triple negative breast cancers. Oncotarget, 5, 1174.

99. Hubbard, R. A., et al. (2011). Cumulative probability of falsepositive recall or biopsy recommendation after 10 years of screening mammography: a cohort study. Annals of Internal Medicine, $155,481$.

100. Wiatrek, R., \& Kruper, L. (2011). Sentinel lymph node biopsy indications and controversies in breast cancer. Maturitas, 69, 7.

101. Cheng, G., Kurita, S., Torigian, D. A., \& Alavi, A. (2011). Current status of sentinel lymph-node biopsy in patients with breast cancer. European Journal of Nuclear Medicine and Molecular Imaging, 38, 562.

102. Zhou, W., et al. (2014). Cancer-secreted miR-105 destroys vascular endothelial barriers to promote metastasis. Cancer Cell, 25, 501.

103. Asaga, S., et al. (2011). Direct serum assay for microRNA-21 concentrations in early and advanced breast cancer. Clinical Chemistry, 57,84 . 
104. Madhavan, D., et al. (2012). Circulating miRNAs as surrogate markers for circulating tumor cells and prognostic markers in metastatic breast cancer. Clinical Cancer Research, 18, 5972.

105. van Schooneveld, E., et al. (2012). Expression profiling of cancerous and normal breast tissues identifies microRNAs that are differentially expressed in serum from patients with (metastatic) breast cancer and healthy volunteers. Breast Cancer Research, 14, R34.

106. Schwarzenbach, H., Milde-Langosch, K., Steinbach, B., Muller, V., \& Pantel, K. (2012). Diagnostic potential of PTEN-targeting miR214 in the blood of breast cancer patients. Breast Cancer Research and Treatment, 134, 933.

107. Eichelser, C., Flesch-Janys, D., Chang-Claude, J., Pantel, K., \& Schwarzenbach, H. (2013). Deregulated serum concentrations of circulating cell-free microRNAs miR-17, miR-34a, miR-155, and miR-373 in human breast cancer development and progression. Clinical Chemistry, 59, 1489.

108. Chen, W., Cai, F., Zhang, B., Barekati, Z., \& Zhong, X. Y. (2013). The level of circulating miRNA-10b and miRNA-373 in detecting lymph node metastasis of breast cancer: potential biomarkers. Tumour Biology, 34, 455.

109. Ahmad, A., et al. (2014). Up-regulation of microRNA-10b is associated with the development of breast cancer brain metastasis. American Journal of Translational Research, 6, 384.

110. Zhao, F. L., et al. (2012). Serum overexpression of microRNA-10b in patients with bone metastatic primary breast cancer. Journal of International Medical Research, 40, 859.

111. Ma, L., Teruya-Feldstein, J., \& Weinberg, R. A. (2007). Tumour invasion and metastasis initiated by microRNA-10b in breast cancer. Nature, 449, 682.

112. Song, S. J., et al. (2013). MicroRNA-antagonism regulates breast cancer stemness and metastasis via TET-family-dependent chromatin remodeling. Cell, 154, 311.

113. Huang, Q., et al. (2008). The microRNAs miR-373 and miR-520c promote tumour invasion and metastasis. Nature Cell Biology, 10, 202.

114. Yan, L. X., et al. (2008). MicroRNA miR-21 overexpression in human breast cancer is associated with advanced clinical stage, lymph node metastasis and patient poor prognosis. $R N A, 14,2348$.
115. Shivapurkar, N., et al. (2014). Recurrence of early stage colon cancer predicted by expression pattern of circulating microRNAs. PloS One, 9, e84686.

116. Kjersem, J. B., et al. (2014). Plasma microRNAs predicting clinical outcome in metastatic colorectal cancer patients receiving first-line oxaliplatin-based treatment. Molecular Oncology, 8, 59.

117. Wang, L. G., \& Gu, J. (2012). Serum microRNA-29a is a promising novel marker for early detection of colorectal liver metastasis. Cancer Epidemiology, 36, e61.

118. Toiyama, Y., et al. (2014). Serum miR-200c is a novel prognostic and metastasis-predictive biomarker in patients with colorectal cancer. Annals of Surgery, 259, 735.

119. Gonzales, J. C., et al. (2011). Comparison of circulating microRNA 141 to circulating tumor cells, lactate dehydrogenase, and prostatespecific antigen for determining treatment response in patients with metastatic prostate cancer. Clinical Genitourinary Cancer, 9, 39.

120. Zhang, H. L., et al. (2013). An elevated serum miR-141 level in patients with bone-metastatic prostate cancer is correlated with more bone lesions. Asian Journal of Andrology, 15, 231.

121. Nguyen, H. C., et al. (2013). Expression differences of circulating microRNAs in metastatic castration resistant prostate cancer and low-risk, localized prostate cancer. Prostate, 73, 346.

122. Zhao, S., Yao, D., Chen, J., \& Ding, N. (2013). Circulating miRNA20a and miRNA-203 for screening lymph node metastasis in early stage cervical cancer. Genetic Testing and Molecular Biomarkers, $17,631$.

123. Kim, S. Y., et al. (2013). Validation of circulating miRNA biomarkers for predicting lymph node metastasis in gastric cancer. Journal of Molecular Diagnostics, 15, 661.

124. Chen, J., et al. (2013). Serum microRNA expression levels can predict lymph node metastasis in patients with early-stage cervical squamous cell carcinoma. International Journal of Molecular Medicine, 32, 557.

125. Dai, X., Chen, A., \& Bai, Z. (2014). Integrative investigation on breast cancer in ER, PR and HER2-defined subgroups using mRNA and miRNA expression profiling. Science Reports, 4, 6566. 